\title{
Nanobundles structural Co-HKUST on the foamed nickel with a high supercapacitor performance
}

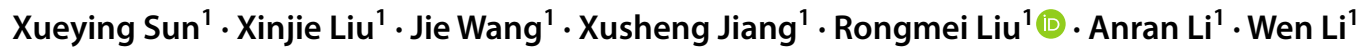

Received: 14 November 2019 / Accepted: 6 February 2020 / Published online: 14 February 2020

(c) Springer Nature Switzerland AG 2020

\begin{abstract}
In this work, we first successfully loaded the MOF structure Co-HKUST nanobundles on a foamed nickel without adding any binder. The Co-HKUST is a pseudo-capacitive material with excellent electrochemical performance as a unique energy storage material. As an electro-active material, such cobalt-based MOF exhibits superior pseudo-capacitive behavior in the $\mathrm{KOH}$ aqueous electrolyte with a high specific capacitance of $578.6 \mathrm{~F} \mathrm{~g}^{-1}$ at $1 \mathrm{~A} \mathrm{~g}^{-1}$, and the specific capacitance remains $452.3 \mathrm{~F} \mathrm{~g}^{-1}$ when the current density increased to $10 \mathrm{~A} \mathrm{~g}^{-1}$ with a good rate performance (78.2\%). Meanwhile, the cycle retention rate of the Co-HKUST is $88.7 \%$ after 1000 cycles at $7.5 \mathrm{~A} \mathrm{~g}^{-1}$. The two-electrode asymmetric supercapacitor (ASC) device is also assembled in this work. The specific capacitance of Co-HKUST//AC ASC is $200.5 \mathrm{~F} \mathrm{~g}^{-1}$ at $1 \mathrm{~A} \mathrm{~g}^{-1}$. Though 5000 cycles at $5 \mathrm{~A} \mathrm{~g}^{-1}$, only $8.2 \%$ of the capacitance is lost. Energy density of asymmetric Co-HKUST//AC device can reach $71.35 \mathrm{Wh} \mathrm{kg}^{-1}$ at $1 \mathrm{~A} \mathrm{~g}^{-1}$ with a power of $809.9 \mathrm{~W} \mathrm{~kg}^{-1}$. More interestingly, even if the current density increased to $10 \mathrm{~A} \mathrm{~g}^{-1}$, the energy density maintains $44 \mathrm{Wh} \mathrm{kg}^{-1}$ at power density of $8.5 \mathrm{~kW} \mathrm{~kg}^{-1}$. The above test results can fully show that the synthesized Co-HKUST material is a promising energy storage material.
\end{abstract}

Keywords Co-HKUST · Asymmetric supercapacitor · Cycling performance · Energy density

\section{Introduction}

Energy exhaustion has prompted more and more researchers to study how to better manage and store energy [1]. People are advancing the concept of renewable energy, looking for better technology, and researching the storage of renewable energy at faster speeds. Supercapacitors, batteries, fuel cells, etc., are the most important electrochemical energy storage devices [2-4]. Among them, as a portable energy storage system, supercapacitors (SCs) stand out, with many unique advantages, such as long cycle capability, high-power output, excellent reversibility and a wide operating temperature range $[5,6]$. In the past few years, due to its potential application in portable electronic devices, research on the new flexible supercapacitors has become one of the most focuses in the field of electrical energy storage [7]. Carbon materials, conductive polymers and transition metal oxides are conventional electrode materials for supercapacitors [8-14]. However, the rapid development of high-performance supercapacitors limits the application of traditional materials. Therefore, the development of new high-performance supercapacitor electrode materials is imminent [15].

Metallic organic framework (MOF) materials has become the darling of supercapacitor materials because of its many special properties, such as high accessible surface areas, tunable pore sizes, open metal sites and ordered crystalline structures $[16,17]$. There are two main types

Electronic supplementary material The online version of this article (https://doi.org/10.1007/s42452-020-2205-6) contains supplementary material, which is available to authorized users.

Rongmei Liu, liurongmei@ahpu.edu.cn | 'School of Biological and Chemical Engineering, Anhui Polytechnic University, Wuhu 241000, Anhui, People's Republic of China. 
of applications of MOFs in terms of supercapacitors. One is that MOFs are applied as templates for metal oxides $[18,19]$, mixed metal oxides [20, 21], metal nanoparticles [22-24], and porous carbon compounds [25, 26]. Another is that the original MOFs are directly applied as active electrode materials. In fact, in recent years, the direct use of the original MOFs as the active material of supercapacitors has been rapidly developed, which not only expands the application of MOFs materials, but also provides more development prospects for new electrode materials about supercapacitors. For instance, Yang et al. [27] synthesized a layered structure Ni-MOF, which has good specific capacitance $\left(1127 \mathrm{~F} \mathrm{~g}^{-1}\right.$ at $\left.0.5 \mathrm{~A} \mathrm{~g}^{-1}\right)$ and cycling performance. Lee et al. [28] successfully synthesized Co-MOF film with a specific capacitance beyond $206.76 \mathrm{~F} \mathrm{~g}^{-1}$ and only lost $1.5 \%$ after 5000 cycles. Yan et al. [29] synthesized an electrode material Ni-MOF-like accordion showing specific capacitances of $988 \mathrm{~F} \mathrm{~g}^{-1}$ at $1.4 \mathrm{~A} \mathrm{~g}^{-1}$. Liu et al. [30] synthesized a layered Cu-MOF structure with an excellent specific capacitance of $1274 \mathrm{~F} \mathrm{~g}^{-1}$, and it lost $12 \%$ of the capacitance during the cycling. However, the current number of related reports is limited, and the supercapacitor performance of these materials needs to be further studied due to poor conductivity and large steric hindrance [31, 32].

Therefore, in order to develop more kinds of MOF electrode materials and further improve their supercapacitance, it is necessary to design a MOF material with a good structure. Not only does it have a stable structure, but it also has a conductive network frame, which ensures a capacitive process with easy electrolyte diffusion and rapid electron transfer and increases the chances of achieving enhanced energy storage performance. Recently, the Behera project from India has synthesized a new MOF structure, which brings some ideas for our research [33]. Co-HKUST (Hong Kong University of Science and Technology) is a new MOF made up of cobalt nodes with 1,3,5-benzenetricarboxylic acid struts between them. The Co-HKUST has the same structure as CU-HKUST-1 and has a high degree of stability. The application of energy storage is one of the important applications of MOF materials, and it is also one of the most studied properties in the performance of MOF materials. Co-HKUST, which has the same structure as CU-HKUST-1, should have the same excellent electrochemical performance as Cu-HKUST-1, but its electrochemical performance needs to be studied urgently.

Herein, we first successful loaded the Co-HKUST nanobundles on the foamed nickel via a simple hydrothermal method and then applied to supercapacitors. The Co-HKUST nanobundles are similar in structure to CU-HKUST, providing good electron and ion transport pathways for the storage of electrolyte ions. In addition, the Co-HKUST nanobundles loaded on the foamed nickel can effectively increase the electrical conductivity of
Co-HKUST and enhance the specific surface area. Meanwhile, the asymmetric supercapacitors (Co-HKUST//Activated carbon ASCs) were assembled. The Co-HKUST// activated carbon (AC) devices had an excellent specific energy of $71.3 \mathrm{Wh} \mathrm{kg}^{-1}$ at a specific power of $809.9 \mathrm{~W} \mathrm{~kg}^{-1}$. Even more interesting is that Co-HKUST//AC ASCs exhibit a good cycling performance at 5000 cycles. All the results of tests show that the Co-HKUST nanobundles are promising and innovative energy storage materials.

\section{Experimental}

\subsection{Synthesis of Co-HKUST nanobundles}

All drugs were obtained from Aladdin Shanghai without further purification. The porosity of the foamed nickel is $95-98 \%$, the thickness is $1.7 \mathrm{~mm}$, and the purity is $99.9 \%$. The foamed nickel used was cleaned before the experiment, and ultrasonically washed with acetone, $1 \mathrm{M} \mathrm{HCl}$ and ethanol in that order. $0.0184 \mathrm{~g}$ of 1,3,5-benzenetricarboxylic acid (BTC) was dissolved in $35 \mathrm{ml}$ of water and $35 \mathrm{ml}$ of ethanol, and stirred for $1 \mathrm{~h}$, after which $0.002 \mathrm{~mol}$ of cobalt chloride was added and dispersed for $1 \mathrm{~h}$. The previously treated foamed nickel was cut into some $1 \times 5 \mathrm{~cm}$ strips into a polytetrafluoroethylene reactor, and the dispersed reaction solution reacted at $120^{\circ} \mathrm{C}$ during $16 \mathrm{~h}$. The reaction vessel was naturally cooled and the foamed nickel ultrasonicated with distilled water and ethanol for $5 \mathrm{~min}$, respectively. Last, the sample placed in an oven and dried at $60^{\circ} \mathrm{C}$ for spare.

\subsection{Characterization}

The phase analysis of the obtained sample was carried out using a Bruker X-ray powder diffractometer (D8 Fous in Germany, Cu Ka, $\lambda=0.1541 \mathrm{~nm}$ ). The sample was pressed on the sample stage. The morphology and structure of the prepared materials were characterized by a Hitachi S- 4800 field emission scanning electron microscope (SEM). X-ray photoelectron spectroscopy (XPS) was obtained on the ESCALAB 250Xi from Thermo Fisher Scientific with Al Ka radiation. TEM and HRTEM are tested on a copper target by FEI F30 at a voltage of $200 \mathrm{kV}$. Fourier transform infrared (FTIR) transmission spectra were recorded on a Nicolet iS10 IR spectrophotometer. I-V curves were recorded by two-point probe measurement (Keithley 2400 semiconductor parameter) at room temperature. The surface area of the as-synthesized sample is measured by the Brunauer-Emmett-Teller (BET) method and the pore size distribution by the Barrett-Joyner-Halenda (BJH) method on Tristar II 3020 from Micromeritics Instrument Corporation. 


\subsection{Electrochemical measurements}

In the electrolyte of $\mathrm{KOH}$ solution (1 M), a platinum electrode and a saturated calomel electrode (SCE) electrode were used as counter electrode and reference electrode, respectively, and the Co-HKUST was used as working electrode for the three-electrode electrochemical test. The electrochemical performance of the two electrodes was tested using asymmetric supercapacitor assembly, assembled with $6 \mathrm{M} \mathrm{KOH}$ as the electrolyte. In the two-electrode test system, the positive electrode is Co-HKUST and the activated carbon is the negative electrode to prepare an aqueous asymmetric supercapacitor (Co-HKUST//AC). All cyclic voltammetry (CV), constant current charge and discharge (GCD), alternating current impedance (EIS) and cycle were performed on the Shanghai Chenhua CHI660E electrochemical workstation. The asymmetric supercapacitor (Co-HKUST//AC) was tested on Wuhan Lan He CT2001A.

\section{Results and discussion}

A schematic of the Co-HKUST synthesis is given in Fig. 1. Two sheets of the foamed nickel were added to the pink solution in which 1,3,5-benzenetricarboxylic acid (BTC) and cobalt chloride were dissolved. The crystal structure of Co-HKUST is composed of $\mathrm{CO}_{2}(\mathrm{COO})_{4}$ units during the high temperature hydrothermal reaction. Each $\mathrm{Co}_{2}(\mathrm{COO})_{4}$ unit is locked by four carboxyl groups from four equivalent BTC molecules, and each BTC molecule is linked to three $\mathrm{CO}_{2}(\mathrm{COO})_{4}$ units to form a Co-HKUST three-dimensional framework during the hydrothermal reaction [33].
The XRD characterization of the samples is shown in Fig. 2a. It can be seen from the XRD pattern that the peaks of the samples are at $12.5,9.2,7.6,25.3,44.5,51.9$, and 76.4, while the peaks of $44.5,51.9$, and 76.4 are the characteristic peaks of the foamed nickel. Other peaks are each comparable to the Co-HKUST characteristic peak. Additionally, the Co-HKUST was characterized by infrared in Fig. $2 \mathrm{~b}$. The peaks at $3428 \mathrm{~cm}^{-1}$ and $1637 \mathrm{~cm}^{-1}$ are respectively due to the $\mathrm{O}-\mathrm{H}$ and $\mathrm{H}-\mathrm{O}-\mathrm{H}$ stretching peaks in the water, with the presence of peaks at $1104 \mathrm{~cm}^{-1}$ and $760 \mathrm{~cm}^{-1}$ corresponding to the $\mathrm{C}=\mathrm{C}$ of aromatic benzene. Further, the other two peaks at $1430 \mathrm{~cm}^{-1}$ and $1366 \mathrm{~cm}^{-1}$ can be indexed as the presence of $\mathrm{Co}-\mathrm{O}$ and $-\mathrm{COO}^{-}$bonds, and the peak at $721 \mathrm{~cm}^{-1}$ is a meta-substitutional absorption peak of the benzene ring which further confirms the formation of the Co-HKUST. These results are consistent with previous research results [32, 33].

The SEM images of the Co-HKUST are shown in Fig. 3a, with the information on the nanobundles structure on the substrate of the foamed nickel. It can be shown on the low-magnification images that Co-HKUST nanobundles grows more uniformly on the surface of the foamed nickel, and the loose structure remains good with uniform size in Fig. 3b. The smooth surface of the nanobundles can be clearly seen under the high-magnification electron microscope. The morphology is close to the straw bundle. Meanwhile, it can be found that the diameter of Co-HKUST is approximately between 50 and $100 \mathrm{~nm}$ from Fig. 3c. Additionally, the Brunauer-Emmett-Teller (BET) test can also prove. The $\mathrm{N}_{2}$ adsorption-desorption isotherms at $77 \mathrm{~K}$ show that Co-HKUST possesses a large BET surface area of around $20.0777 \mathrm{~m}^{2} \mathrm{~g}^{-1}$ and a large number of mesopores

Fig. 1 Synthetic schematic of the Co-HKUST
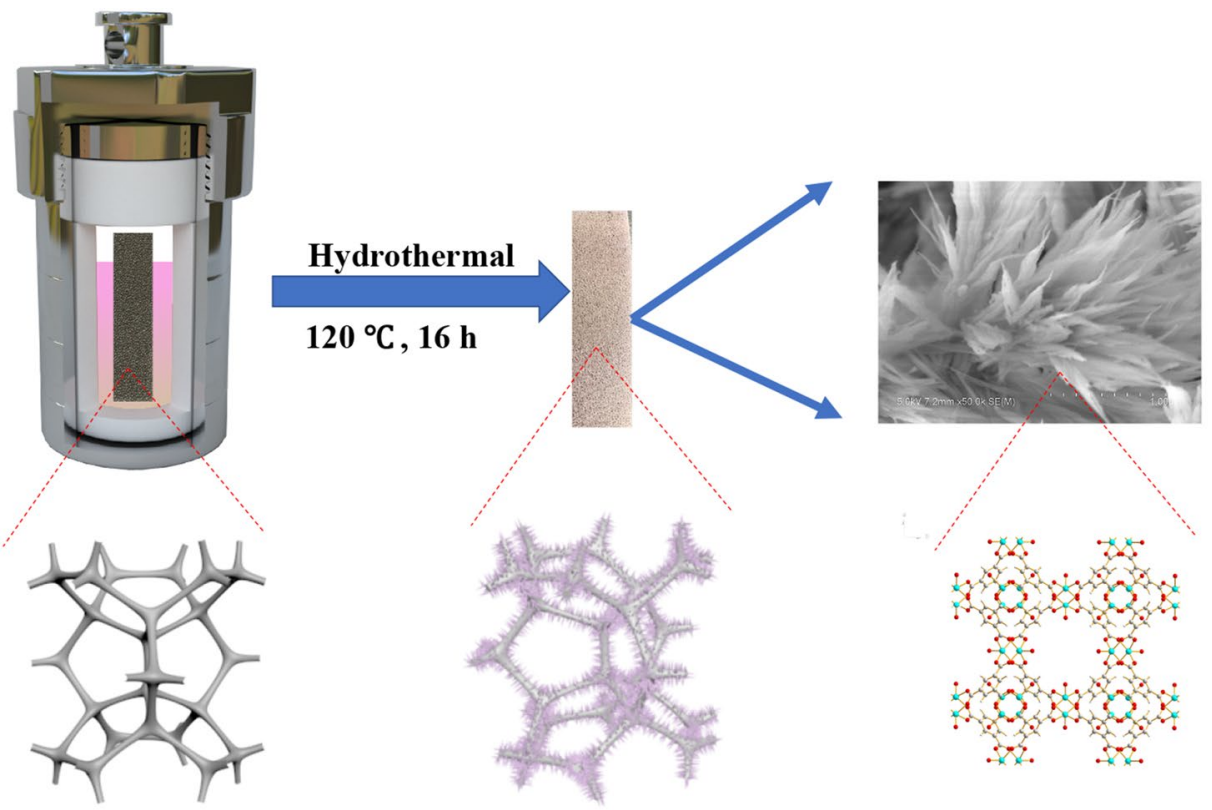

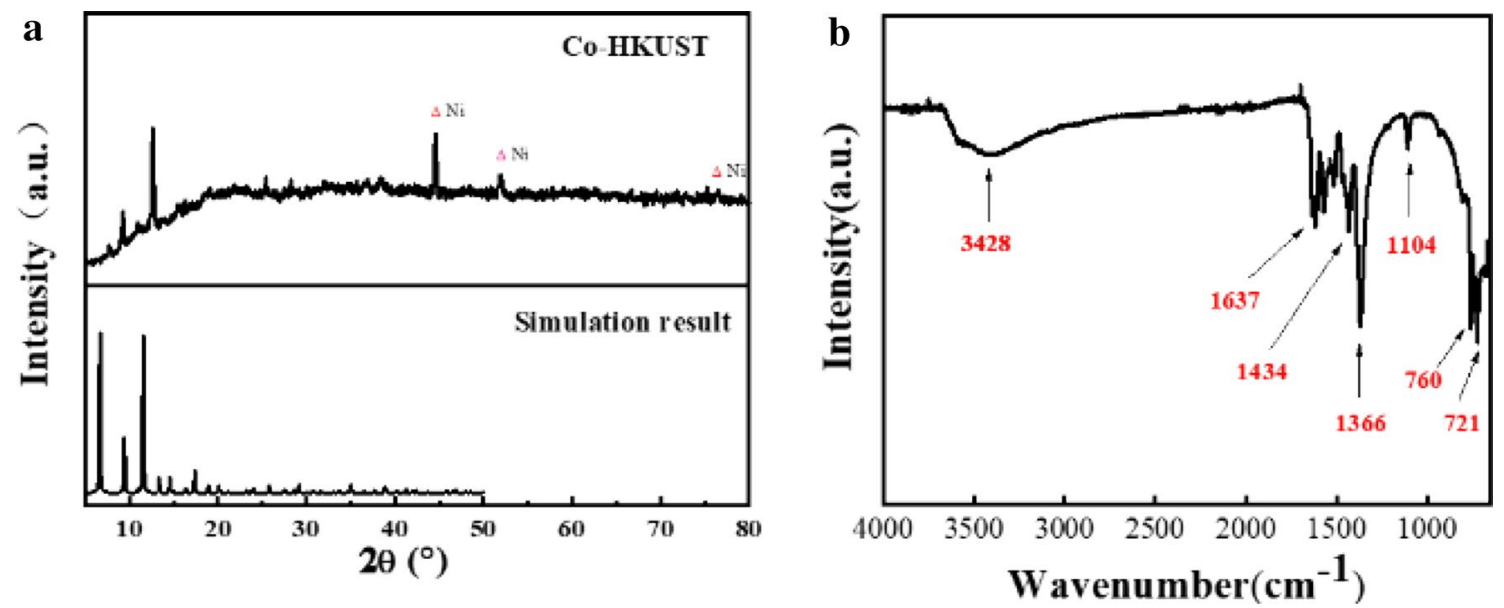

Fig. 2 a XRD pattern and b FTIR spectra of Co-HKUST

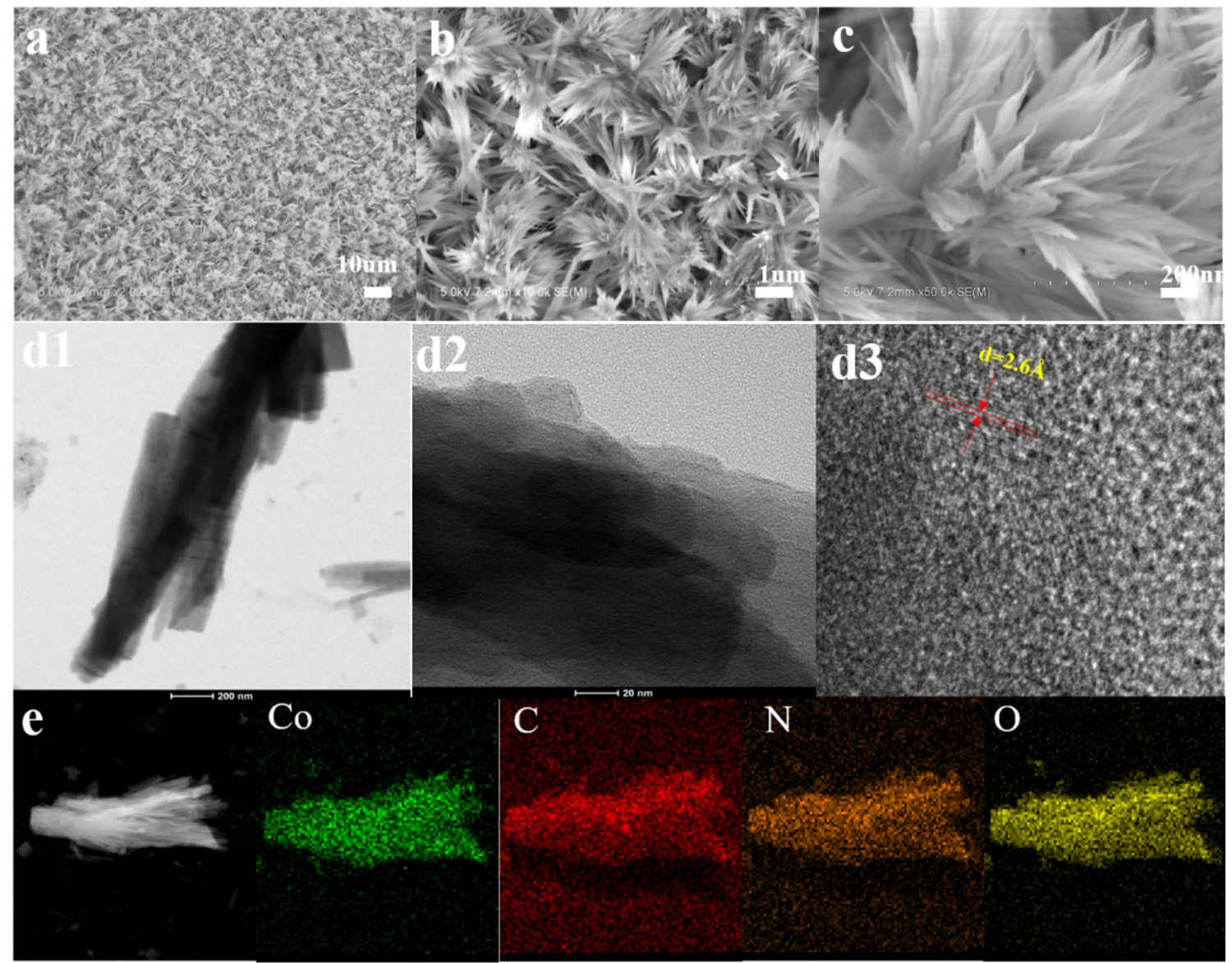

Fig. 3 a-c Different multiplication of SEM images, $\mathbf{d}$ TEM images and HRTEM images, e element mapping of Co-HKUST

with average pore diameter at about $2-5 \mathrm{~nm}$ in Fig. S1a, $1 \mathrm{~b}$. The $\mathrm{N}_{2}$ adsorption-desorption isotherms and pore size distribution of the foamed nickel are also exhibited in Fig. S1c, 1d. The results show that the presence of the
Co-HKUST enhances the specific surface area. To evaluate the electrical behavior of Co-HKUST, the $I-V$ responses were measured at room temperature in Fig. S2. The conductance of the pure foamed nickel and the Co-HKUST

\section{SN Applied Sciences}


are, respectively, $261.9 \pm 0.1 \mathrm{mS}$ and $696.5 \pm 0.3 \mathrm{mS}$. Due to the growth of the Co-HKUST structure on the foamed nickel, the conductance becomes larger and the resistance becomes smaller, thereby having better electrochemical performance.

TEM analysis also confirmed its nanobundles characteristics in Fig. 3d. The HRTEM image exhibits well-resolved lattice fringes, revealing good crystallization characteristics of the Co-HKUST in Fig. 3d3. This is consistent with the XRD pattern. Meanwhile, the element mapping of the CoHKUST nanobundles is characterized in Fig. 3e. In Fig. 3e, it can be seen that all elements of the Co-HKUST are distributed over the sample and are more evenly distributed. In a word, it can be found that $\mathrm{Co}$ is mainly distributed on the sample, and $\mathrm{C}, \mathrm{N}, \mathrm{O}$, which are distributed in the air, are distributed on the sample and the substrate. The results of TEM were consistent with results of the surface elemental samples, confirming the successful synthesis of the Co-HKUST.
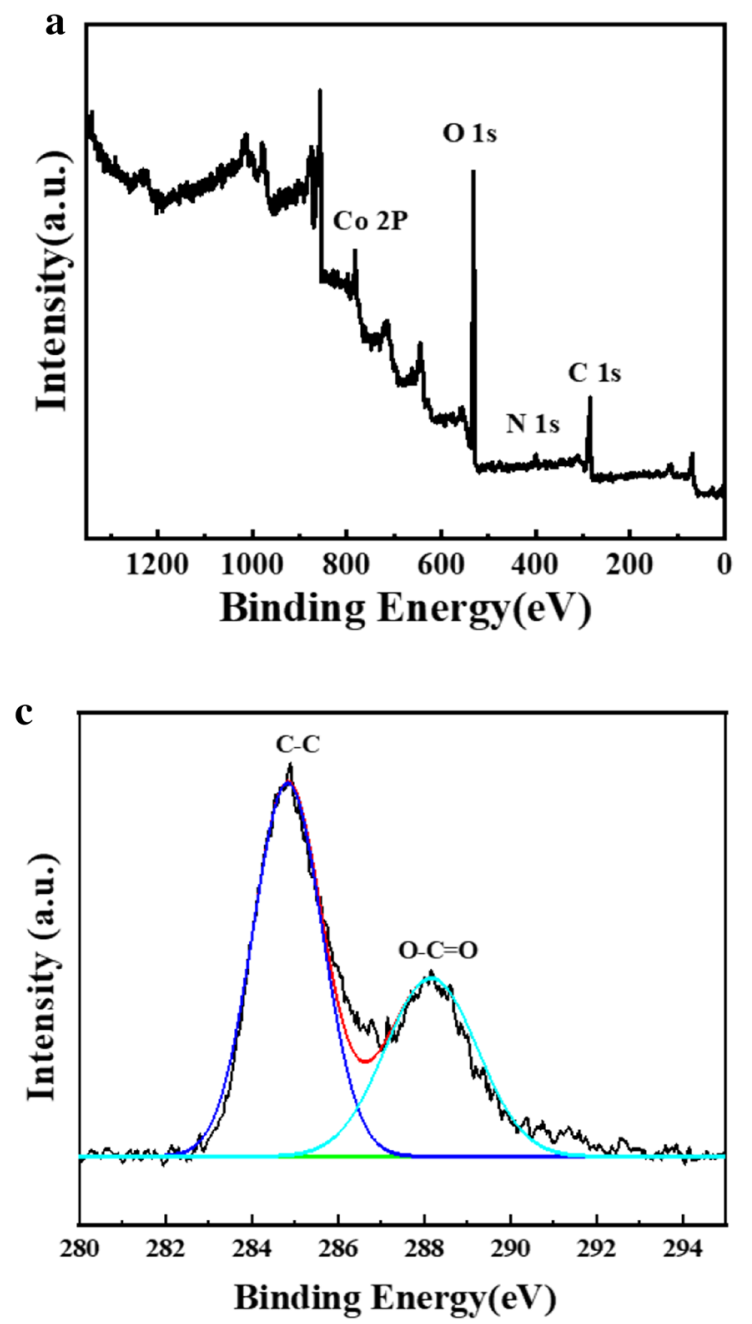

The surface chemical state of the prepared Co-HKUST and the elemental composition were confirmed by XPS measurement. It can be found that cobalt, carbon and oxygen are distributed on the surface of the Co-HKUST in Fig. $4 a$. In the element spectrum of cobalt in Fig. $4 b$, the peaks at six positions correspond well to $\operatorname{Co} 2 p_{1 / 2}$, $\operatorname{Co} 2 p_{3 / 2}$, and the position of the satellite peak, which proves the presence of $\mathrm{Co}$ (II) and Co(III) in the Co-HKUST [34-36]. In the $C 1 s$ spectrum in Fig. 4c, the peak at $284.84 \mathrm{eV}$ corresponds to $\mathrm{C}-\mathrm{C}$, and $288.14 \mathrm{eV}$ corresponds to $\mathrm{O}-\mathrm{C}=\mathrm{O}$ bond, and the results are consistent with the structural skeleton of Co-HKUST [35, 37, 38]. In the spectrum of O1s in Fig. $4 \mathrm{~d}$, the peaks at $531.4 \mathrm{eV}$ and $533.1 \mathrm{eV}$ are consistent with the previously reported $\mathrm{C}-\mathrm{O}$ bond, $\mathrm{O}=\mathrm{C}-\mathrm{O}$ bond, demonstrating the presence of $\mathrm{Co}_{2}(\mathrm{COO})_{4}$ structural units in the Co-HKUST [35, 39]. In summary, all results of test are consistent with the Co-HKUST structure, demonstrating the successful synthesis of the Co-HKUST structure on the foamed nickel.
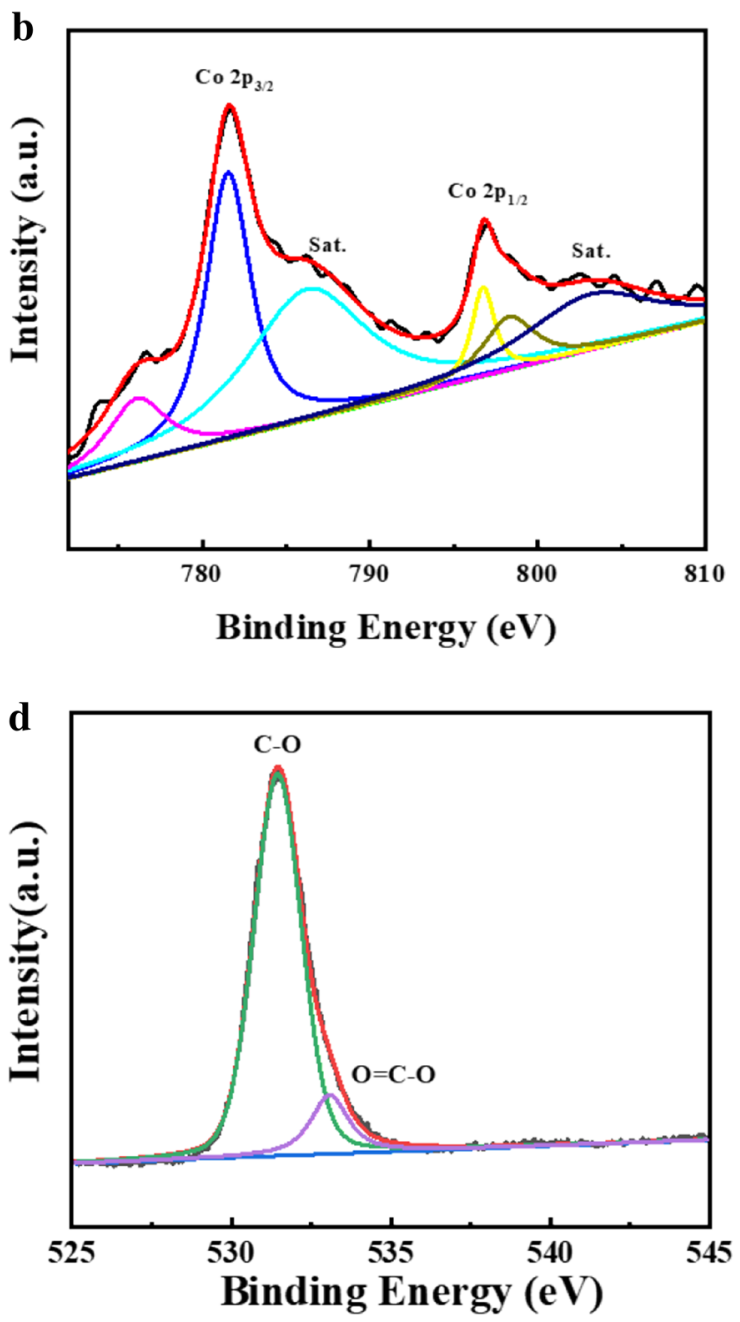

Fig. 4 a Full XPS spectra, b Co, c C, d O of Co-HKUST 
Figure 5a exhibits the electrochemical performance of Co-HKUST through three-electrode tests. The area of the Co-HKUST exposed to the electrolyte is $1 \mathrm{~cm}^{2}$, and the mass is about $2-3 \mathrm{mg}$. From the cyclic voltammogram of Co-HKUST, as the sweep speed increases, the shape of the $\mathrm{CV}$ curve has no clear changes. The shape of the cyclic voltammetry curve does not show the characteristics of pure electric double-layer capacitors (EDLC), and a pair of significant redox absorption peaks appearing between 0.2 and $0.3 \mathrm{~V}$ show a typical pseudo-capacitor type [40]. The possible charge storage mechanisms are in the redox reactions of equations [40-42]:

$\mathrm{Co}_{s}^{\prime \prime}+\mathrm{OH}^{-} \leftrightarrow \mathrm{Co}_{s}^{\prime \prime}(\mathrm{OH})_{a d}+e^{-1}$
$\mathrm{Co}_{s}^{\prime \prime}(\mathrm{OH})_{a d} \leftrightarrow \mathrm{Co}_{s}^{\prime \prime \prime}(\mathrm{OH})_{a d}+e^{-1}$

The capacitance of the material is mainly derived from a pseudo-capacitor based on a redox mechanism. In addition, as the scanning rate increases, the surrounding area of the CV curves increases, indicating that the Co-HKUST material is favorable for the fast Faraday reaction. It can be found that the redox peak can be observed even if the sweep speed is increased to $50 \mathrm{mV} \mathrm{s}^{-1}$, indicating that the material has good rate performance, which is consistent with the test results [32]. In addition, the potentials of the oxidation and reduction peaks are shifted to larger positive and negative values, respectively, which may be mainly due to an increase in the internal resistance of the electrode.

Meanwhile, the galvanostatic charge-discharge (GCD) curves of Co-HKUST nanobundles electrode was further investigated for the chronopotentiometry (CP) curve in Fig. $5 \mathrm{~b}$. There is a significant discharge platform at about $0.2-0.3 \mathrm{~V}$ reflecting the strong pseudo-absorption behavior of the reversible Faraday redox reaction, which is in accordance with the results of the CV curves at all current densities in Fig. 5a. In addition, Co-HKUST powder was synthesized without adding the foamed nickel under the same conditions, and its electrochemical performance was
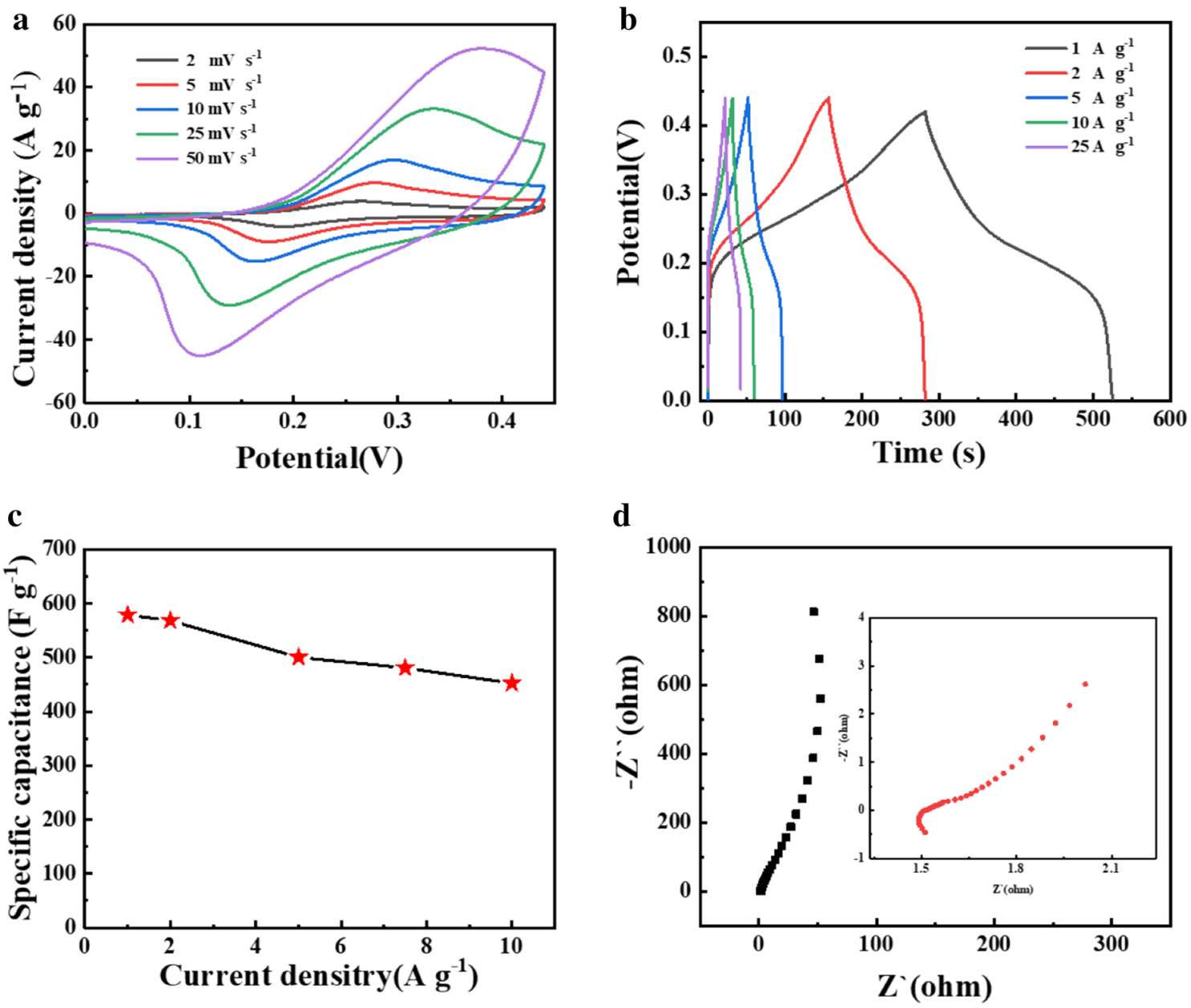

Fig. 5 a Cyclic voltammetry curves at 2, 5, 10, 25, $50 \mathrm{mV} \mathrm{s}^{-1}$, b Galvanostatic charge-discharge curves at 1, 2, 5, 10, 25 A g ${ }^{-1}, \mathbf{c}$ the rate performance, $\mathbf{d}$ electrochemical impedance spectroscopy curve of Co-HKUST 
tested in Fig. S3. The similar redox peaks also appear in the CV curve of Co-HKUST powder in Fig. S3a. Simultaneously, after the calculation, the specific capacitance is $578.6 \mathrm{~F} \mathrm{~g}^{-1}$, 568.2 $\mathrm{F} \mathrm{g}^{-1}, 500.3 \mathrm{~F} \mathrm{~g}^{-1}, 480.7 \mathrm{~F} \mathrm{~g}^{-1}, 452.3 \mathrm{~F} \mathrm{~g}^{-1}$ at $1,2,5$, $7.5,10 \mathrm{~F} \mathrm{~g}^{-1}$, respectively. The rate performance curve is shown in Fig. $5 \mathrm{c}$. It can be found that the electrode material has a large surface area and retains a capacitance of $452.3 \mathrm{~F} \mathrm{~g}^{-1}$ at $10 \mathrm{~A} \mathrm{~g}^{-1}$, indicating that the material has a good rate capability. The rate performance is $78.2 \%$, indicating that the Co-HKUST has a large current charge and discharge capability and is an excellent electrode material. From the GCD curve in Fig. S3b, the specific capacitance of the Co-HKUST powder is $367.4 \mathrm{~F} \mathrm{~g}^{-1}$, which is lower than the Co-HKUST. Because the Co-HKUST powder has added a binder when synthesizing an electrode, its electrochemical performance is reduced. It also proved the superiority of the Co-HKUST growing on the foamed nickel. In order to evaluate the conductivity and charge transport properties at the electrode/electrolyte interface, electrical impedance spectroscopy (EIS) is performed in the frequency range of $0.01 \mathrm{~Hz}$ to $10 \mathrm{kHz}$. The EIS curve of the Co-HKUST composes a semicircle in the high-frequency region and a straight line in the low-frequency region, which indicates that the reaction process of the electrode reaction under steady state conditions is a mixed reaction control process composed of charge transfer and diffusion migration. The EIS curve of the Co-HKUST powder is also shown in Fig. S3c. Generally, Rs refers to the solution resistance, including electrolytic resistance, inherent resistance of the material, and contact resistance between active material and current collector [43]. Rct is a charge transfer resistance existing due to the Faraday reaction. The smaller the semicircle in the high-frequency region, the smaller the charge transfer resistance $[44,45]$. The impedance tests in Fig. 5d show that the Co-HKUST has a small solution resistance and a small ion transfer resistance in $1 \mathrm{M} \mathrm{KOH}$, which is a good electrochemical energy storage material.

One of the more important factors in evaluating the electrochemical performance of electrode materials is the cycle performance. Thus, the cycling performance of $\mathrm{Co}$ HKUST was also tested in $1 \mathrm{M} \mathrm{KOH}$ solution at $7.5 \mathrm{~A} \mathrm{~g}^{-1}$ shown in Fig. 6. At the first lap of the cycling, the specific capacitance of Co-HKUST is $804.7 \mathrm{~F} \mathrm{~g}^{-1}$. In the 1000th lap, the specific capacitance of Co-HKUST is $713.5 \mathrm{~F} \mathrm{~g}^{-1}$, and the retention is $88.7 \%$ of the initial capacitance, which shows the excellent cycling performance. The cycling performance of the Co-HKUST powder was also given in Fig. S3d with less specific capacitance. Additionally, the Co-HKUST after cycling was characterized. The result of SEM was showing that the nanobundles structure did not collapse in Fig. 6a insets. The XRD pattern of the Co-HKUST after cycling is shown in Fig. S4. It is seen that the electrodes cycled retained its structure. Meanwhile, the surface elements have not changed in the EDS test in Fig. 6b. The above results were also the reason for maintaining high retention.

Additionally, an asymmetric supercapacitor Co-HKUST// AC was assembled and electrochemically tested. As for a supercapacitor, the charge balance follows the relationship $Q^{+}=Q^{-}$. The charge stored by each electrode usually depends on specific capacitance $(C)$, the potential range for charge/discharge process and the mass of the electrode $(m)$ following equation: $m^{+} / m^{-}=C^{-} \times V^{-} / C^{+} \times V^{+}$. Therefore, to design the Co-HKUST//AC ASC, loading mass ratio of active materials (m(Co-HKUST)/m(AC)) is 0.38 according to formula $[46,47]$. Figure 7 a shows the CV curve for the Co-HKUST and AC in the two-electrode electrochemical test at a sweep speed of $10 \mathrm{mV} \mathrm{s}^{-1}$. It can be
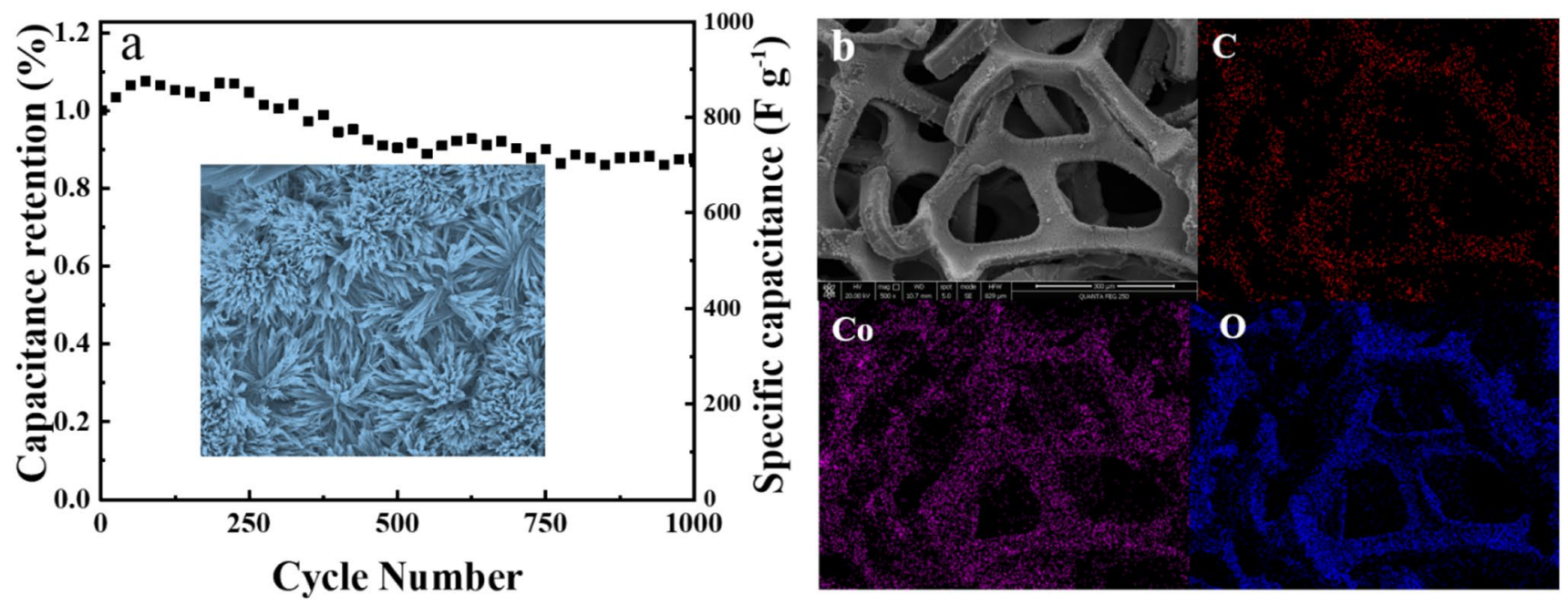

Fig. 6 a Cycle performance of Co-HKUST at $7.5 \mathrm{~A} \mathrm{~g}^{-1}$ (insets: the SEM of the Co-HKUST after cycling), b the EDS of the Co-HKUST after cycling 

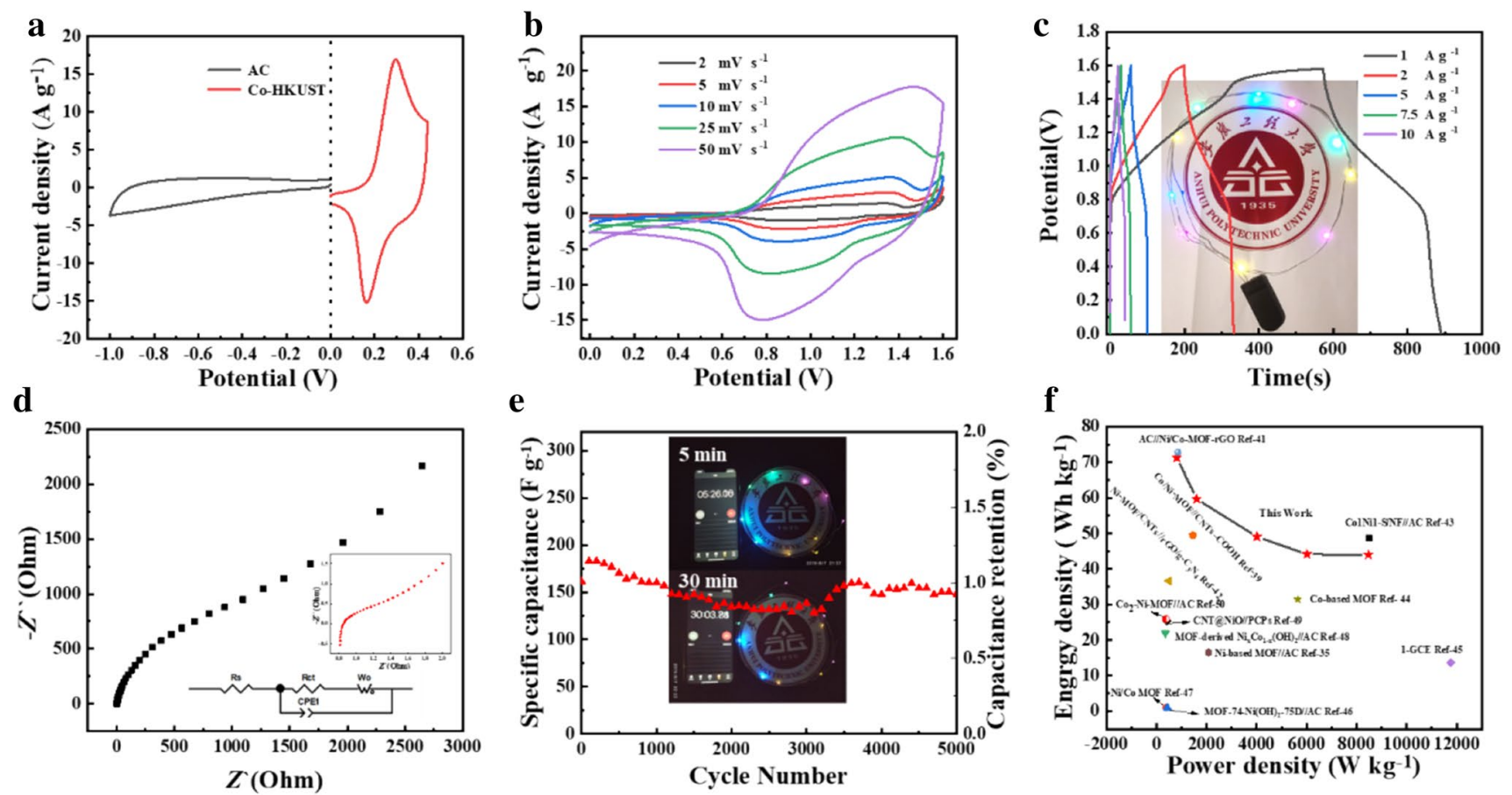

Fig. 7 a Cyclic voltammetry curves of AC and Co-HKUST, b cyclic voltammetry curves at $2,5,10,25,50 \mathrm{mV} \mathrm{s}^{-1}$, c Galvanostatic charge-discharge curves at 1, 2, 5, 7.5, $10 \mathrm{~A} \mathrm{~g}^{-1}$ (inset: LED lights), d electrochemical impedance spectroscopy curves (inset: The equiva-

seen that the $A C$ electrode applies in a potential range of -1 to 0 V, while the Co-HKUST electrode material operates between 0 and $0.44 \mathrm{~V}$. Therefore, the fabricated asymmetrical device can work in the range of $0-1.44 \mathrm{~V}$. However, in practice, we observed that the electrode active material can operate stably within a wide potential window of $1.6 \mathrm{~V}$, so the operating potential window of the device is limited to $1.6 \mathrm{~V}$. CV curves were performed on the ASC apparatus fabricated in Fig. 7b. The samples were tested at different scanning speeds $\left(2,5,10,25,50 \mathrm{mV} \mathrm{s}^{-1}\right)$, and the $\mathrm{CV}$ curves were typical and not deformed at all scanning rates. The pseudo-capacitor characteristics show good rate performance [29]. The constant current discharge curve of the Co-HKUST//AC device at different current densities is shown in Fig. 7c. As shown in Fig. 7c, the Co-HKUST//AC devices reach a high specific capacitance of $200.5 \mathrm{~F} \mathrm{~g}^{-1}$ at $1 \mathrm{~A} \mathrm{~g}^{-1}$. The specific capacitance of Co-HKUST//AC device remains $123.7 \mathrm{~F} \mathrm{~g}^{-1}$ at $10 \mathrm{~A} \mathrm{~g}^{-1}$ with a high rate performance (61.7\%). The EIS curve of Co-HKUST//AC was also composed of a semicircle in the high-frequency region and a straight line in the low frequency region in Fig. $7 \mathrm{~d}$. The analog equivalent circuit that meets the experimental data exhibits a very low $R_{\mathrm{s}}$ of $0.83 \Omega$ and the $R_{\mathrm{ct}}$ of only $1.60 \Omega$ in the Fig. $7 \mathrm{~d}$ inset. These values, as well as the lowfrequency region near the line, exhibited a well supercapacitor performance of the material [48]. lent circuit fitted to the experimental EIS data), e cycling stability of the Co-HKUST//AC (insets: LED lights at $5 \mathrm{~min}$ and $30 \mathrm{~min}$ ) at $10 \mathrm{~A} \mathrm{~g}^{-1}$, f Ragone plot of energy density and power density compared with other reported data

The four Co-HKUST//AC devices were used to illuminate a string of LED lights with $6 \mathrm{~V}$ rated voltage, the string can last more than $30 \mathrm{~min}$. Meanwhile, after 5000 cycles of Co-HKUST//AC devices, the capacitance retention rate reached a staggering $91.8 \%$ in Fig. 7e. After calculation, the Co-HKUST//AC devices reach a high-power density and a high-energy density at $1 \mathrm{~A} \mathrm{~g}^{-1}\left(809.9 \mathrm{~W} \mathrm{~kg}^{-1}\right.$, $71.35 \mathrm{Wh} \mathrm{kg}^{-1}$, respectively). In addition, at a high current density of $10 \mathrm{~A} \mathrm{~g}^{-1}$, the device maintains an energy density of $44.0 \mathrm{Wh} \mathrm{kg}^{-1}$ at $8.5 \mathrm{~kW} \mathrm{~kg}^{-1}$. For comparison, we list the power and energy density of other recently reported electrochemical energy storage material of similar structures. The Co-HKUST is one of the best reported recently in flexible supercapacitors, such as Co/Ni-MOF//CNTs$\mathrm{COOH}$ (49.5 Wh kg-1, $2 \mathrm{~A} \mathrm{~g}^{-1}$ ) [44], AC//Ni/Co-MOF-rGO (72.8 $\mathrm{Wh} \mathrm{kg}^{-1}, 1 \mathrm{~A} \mathrm{~g}^{-1}$ ) [48], Ni-MOF/CNTs//rGO/g- $\mathrm{C}_{3} \mathrm{~N}_{4}$ (36.6 $\mathrm{Wh} \mathrm{kg}^{-1}, 0.5 \mathrm{~A} \mathrm{~g}^{-1}$ ) [49] and so on [40, 50-57].

\section{Conclusions}

In this work, the Co-HKUST nanobundles were first loaded on the foamed nickel and used it as an electrical material for supercapacitors. The prepared Co-HKUST nanobundles have excellent electrochemical properties. After testing, the material has a specific capacitance of 578.6 in $1 \mathrm{~A} \mathrm{~g}^{-1}$. 
When the current density is $10 \mathrm{~A} \mathrm{~g}^{-1}$, the specific capacitance is $452.3 \mathrm{~F} \mathrm{~g}^{-1}$, and the rate performance is amazing $78.2 \%$. After 1000 cycles at $7.5 \mathrm{~A} \mathrm{~g}^{-1}$, the cycle retention rate of Co-HKUST nanobundles is $88.7 \%$. When assembled with an asymmetric supercapacitor (Co-HKUST// $\mathrm{AC})$, the specific capacitance is $200.5 \mathrm{~F} \mathrm{~g}^{-1}$ at $1 \mathrm{~A} \mathrm{~g}^{-1}$. The capacitance retention can keep a staggering $91.8 \%$ after 5000 cycles at $5 \mathrm{~A} \mathrm{~g}^{-1}$. In addition, at current density of $1 \mathrm{~A} \mathrm{~g}^{-1}$, the energy density is $71.35 \mathrm{Wh} \mathrm{kg}^{-1}$ at power of $809.9 \mathrm{~W} \mathrm{~kg}^{-1}$. When the current increased to 10 times, the energy density can still be kept up to $44.0 \mathrm{Wh} \mathrm{kg}^{-1}$ at $8.5 \mathrm{~kW} \mathrm{~kg}^{-1}$. In summary, the Co-HKUST is an excellent electrochemical material with unlimited potential for use in materials science.

Acknowledgements This work was supported by the National Natural Science Foundation of China (No. 21301002) and the University Natural Science Research Project of Anhui Province (No. KJ2018A0107).

\section{Compliance with ethical standards}

Conflict of interest The authors declare that they have no conflict of interest.

\section{References}

1. Xu XL, Shi WH, Li P, Ye SF, Ye CZ, Ye HJ, Lu TM, Zheng AA, Zhu JX, Xu LX, Zhong MQ, Cao XH (2017) Facile fabrication of threedimensional graphene and metal-organic framework composites and their derivatives for flexible all-solid-state supercapacitors. Chem Mater 29:6058-6065

2. Morozan A, Jaouen F (2012) Metal organic frameworks for electrochemical applications. Energy Environ Sci 5:9269-9290

3. Wang L, Han YZ, Feng X, Zhou JW, Qi PF, Wang B (2016) Metalorganic frameworks for energy storage: Batteries and supercapacitors. Coord Chem Rev 307:361-381

4. Fan L, Tang L, Gong HF, Yao ZH, Guo R (2012) Carbon-nanoparticles encapsulated in hollow nickel oxides for supercapacitor application. J Mater Chem 22:16376-16381

5. Simon P, Gogotsi $Y$ (2008) Materials for electrochemical capacitors. Nat Mater 7:845-854

6. Jiao Y, Liu Y, Yin BS, Zhang SW, Qu FY, Wu X (2014) Hybrid $\mathrm{a}-\mathrm{Fe}_{2} \mathrm{O}_{3} @ \mathrm{NiO}$ heterostructures for flexible and high performance supercapacitor electrodes and visible light driven photocatalysts. Nano Energy 10:90-98

7. He YM, Chen WJ, Li XD, Zhang ZX, Fu JC, Zhao CH, Xie EQ (2012) Freestanding three-dimensional graphene $/ \mathrm{MnO}_{2}$ composite networks as ultralight and flexible supercapacitor electrodes. ACS Nano 7:174-182

8. Wang KX, Zhang JN, Xia W, Zou RQ, Guo JH, Gao ZM, Yan WF, Guo SJ, Xu Q (2015) Dual templating route to three-dimensional ordered mesoporous carbon nanonetworks: tuning the mesopore type for electrochemical performance optimization. J Mater Chem A 3:18867-18873

9. Zhu Y, Murali S, Stoller MD, Ganesh KJ, Cai W, Ferreira PJ, Pirkle A, Wallace RM, Cychosz KA, Thommes M, Su D, Stach EA, Ruoff RS (2011) Carbon-based supercapacitors produced by activation of graphene. Science 332:1537-1541
10. Faraji S, Ani FN (2014) Microwave-assisted synthesis of metal oxide/hydroxide composite electrodes for high power supercapacitors-a review. J Power Sources 263:338-360

11. Snook GA, Kao P, Best AS (2011) Conducting-polymer-based supercapacitor devices and electrodes. J Power Sources 196:1-12

12. Bi Z, Kong Q, Cao Y, Sun G, Su F, WeiX LX, Ahmad A, Xie L, Chen CM (2019) Biomass-derived porous carbon materials with different dimensions for supercapacitor electrodes: a review. J Mater Chem A 7:16028-16045

13. Gharahcheshmeh MH, Gleason KK (2019) Device fabrication based on oxidative chemical vapor deposition (oCVD) synthesis of conducting polymers and related conjugated organic materials. Adv Mater Interfaces 6:1801564

14. An CH, Zhang Y, Guo HN, Wang YJ (2019) Metal oxide-based supercapacitors: progress and prospective. Nanoscale Adv 1:4644-4658

15. Yang J, Zheng C, Xiong PX, Li YF, Wei MD (2014) Zn-doped NiMOF material with a high supercapacitive performance. J Mater Chem A 2:19005-19010

16. Furukawa H, Cordova KE, O'Keeffe M, Yaghi OM (2013) The chemistry and applications of metal-organic frameworks. Science 341:1230444-1230457

17. Liu XX, Shi CD, Zhai CW, Cheng ML, Liu Q, Wang GX (2016) Cobalt-based layered metal-organic framework as an ultrahigh capacity supercapacitor electrode material. ACS Appl Mater Interfaces 8:4585-4591

18. Hall AS, Kondo A, Maeda K, Mallouk TE (2013) Microporous Brookite-phase titania made by replication of a metal-organic framework. J Am Chem Soc 135:16276-16279

19. Kimitsuka Y, Hosono E, Ueno S, Zhou HS, Fujihara S (2013) Fabrication of porous cubic architecture of $\mathrm{ZnO}$ using $\mathrm{Zn}$-terephthalate MOFs with characteristic microstructures. Inorg Chem 52:14028-14033

20. Zhao J, Zhang YL, Su PP, Jiang ZX, Yang QH, Li C (2013) Preparation of $\mathrm{Zn}-\mathrm{Co}-\mathrm{O}$ mixed-metal oxides nanoparticles through a facile coordination polymer based process. RSC Adv 3:4081-4085

21. Cui HJ, Shi JW, Yuan BL, Fu ML (2013) Synthesis of porous magnetic ferrite nanowires containing $\mathrm{Mn}$ and their application in water treatment. J Mater Chem A 1:5902-5907

22. He LC, Liu Y, Liu JZ, Xiong YS, Zheng JZ, Liu YL, Tang ZY (2013) Effective chiral discrimination of tetravalent polyamines on the compaction of single DNA molecules. Angew Chem Int Ed 52:3741-3745

23. He LC, Liu Y, Liu JZ, Xiong YS, Zheng JZ, Liu YL, Tang ZY (2013) Effective chiral discrimination of tetravalent polyamines on the compaction of single DNA molecules. Angew Chem 125:3829-3833

24. Aijaz A, Akita T, Tsumori N, Xu Q (2013) Metal-organic framework-immobilized polyhedral metal nanocrystals: reduction at solid-gas interface, metal segregation, core-shell structure, and high catalytic activity. J Am Chem Soc 135:16356-16359

25. Xia W, Qiu B, Xia DG, Zou RQ (2013) Facile preparation of hierarchically porous carbons from metal-organic gels and their application in energy storage. Sci Rep 3:1935

26. Zhang P, Sun F, Xiang ZH, Shen ZG, Yun J, Cao DP (2014) ZIFderived in situ nitrogen-doped porous carbons as efficient metal-free electrocatalysts for oxygen reduction reaction. Energy Environ Sci 7:442-450

27. Yang J, Xiong PX, Zheng C, Qiu H, Wei MD (2014) Metalorganic frameworks: a new promising class of materials for a high performance supercapacitor electrode. J Mater Chem A 2:16640-16644

28. Lee DY, Yoon SJ, Shrestha NK, Lee SH, Ahn H, Han SH (2012) Supercapacitive property of metal-organic-frameworks with 
different pore dimensions and morphology. Microporous Mesoporous Mater 153:163-165

29. Yan $Y$, Gu P, Zheng SS, Zheng MB, Pang H, Xue HG (2016) Facile synthesis of accordion-like Ni-MOF superstructure for high-performance flexible supercapacitors. J Mater Chem A 4:19078-19085

30. Liu Q, Liu X, Shi C, Zhang Y, Feng X, Cheng ML, Su S, Gu J (2015) A copper-based layered coordination polymer: synthesis, magnetic properties and electrochemical performance in supercapacitors. Dalton Trans 44:19175-19184

31. Morozan A, Jaouen F (2012) Metal organic frameworks for electrochemical applications. Energy Environ Sci 5:9269

32. Yang J, Ma ZH, Gao WX, Wei MD (2016) Layered structural Cobased MOF with conductive network frames as a new supercapacitor electrode. Chem Eur J 23:631-636

33. Marri SR, Chauhan N, Tiwari RK, Kumar J, Behera JN (2018) Two novel 3D-MOFs (Ca-TATB and Co-HKUST): synthesis, structure and characterization. Inorg Chim Acta 47:8-14

34. Zhang YF, Ouyang SX, Yu Q, Ye JH (2015) Modulation of sulfur partial pressure in sulfurization to significantly improve photoelectrochemical performance over $\mathrm{Cu}_{2} \mathrm{ZnSnS}_{4}$ photocathode. Chem Commun 51:14057-14059

35. Gu WL, Hu LY, Hong W, Jia XF, Li J, Wang E (2016) Noble-metalfree $\mathrm{CO}_{3} \mathrm{~S}_{4}-\mathrm{S} / \mathrm{G}$ porous hybrids as an efficient electrocatalyst for oxygen reduction reaction. Chem Sci 7:4167-4173

36. Du J, Zhang T, Xing JL, Xu CL (2017) Hierarchical porous $\mathrm{Fe}_{3} \mathrm{O}_{4} /$ $\mathrm{CO}_{3} \mathrm{~S}_{4}$ nanosheets as efficient electrocatalysts for oxygen evolution reaction. J Mater Chem A 5:9210-9216

37. Chen Y, Li J, Mei T, Hu XG, Liu DW, Wang JC, Hao M, Li JH, Wang JY, Wang XB (2014) Low-temperature and one-pot synthesis of sulfurized graphene nanosheets via in situ doping and their superior electrocatalytic activity for oxygen reduction reaction. J Mater Chem A 2:20714-20722

38. Wang K, Dong XM, Zhao CJ, Qian XZ, Xu YL (2015) Facile synthesis of $\mathrm{Cu}_{2} \mathrm{O} / \mathrm{CuO} / \mathrm{RGO}$ nanocomposite and its superior cyclability in supercapacitor. Electrochim Acta 152:433-442

39. Foo CY, Sumboja A, Tan DJH, Wang J, Lee PS (2014) Flexible and highly scalable $\mathrm{V}_{2} \mathrm{O}_{5}-\mathrm{rGO}$ electrodes in an organic electrolyte for supercapacitor devices. Adv Energy Mater 4:1400236

40. Kang L, Sun SX, Kong LB, Lang JW, Luo YC (2014) Investigating metal-organic framework as a new pseudo-capacitive material for supercapacitors. Chin Chem Lett 25:957-961

41. Liu XX, Shi CD, Zhai CW, Cheng ML, Liu Q, Wang GW (2016) Cobalt-based layered metal-organic framework as an ultrahigh capacity supercapacitor electrode material. ACS Appl Mater Interfaces 8:4585-4591

42. Sun X, Wang GK, Hwang JY, Lian J (2011) Porous nickel oxide nano-sheets for high performance pseudocapacitance materials. J Mater Chem 21:16581-16588

43. Paravannoor A, Nair SV, Pattathil P, Manca M, Balakrishnan A (2015) High voltage supercapacitors based on carbon-grafted $\mathrm{NiO}$ nanowires interfaced with an aprotic ionic liquid. Chem Commun 51:6092-6095

44. Jiao Y, Pei J, Chen DH, Yan CS, Hu YY, Zhang Q, Chen G (2017) Mixed-metallic MOF based electrode materials for high performance hybrid supercapacitors. J Mater Chem A 5:1094-1102
45. Hassan S, Suzuki M, Mori S, El-Moneim AA (2014) $\mathrm{MnO}_{2}$ /carbon nanowall electrode for future energy storage application: effect of carbon nanowall growth period and $\mathrm{MnO}_{2}$ mass loading. RSC Adv 4:20479-20488

46. Gp X, Pg He, Huang BY, Chen TF, Bo Z, Fisher TS (2017) Graphene nanopetal wire supercapacitors with high energy density and thermal durability. Nano Energy 38:127-136

47. Wu X, Yao SY (2017) Flexible electrode materials based on $\mathrm{WO}_{3}$ nanotube bundles for high performance energy storage devices. Nano Energy 42:143-150

48. Rahmanifar MS, Hesari $H$, Noori A, Masoomi MY, Morsali A, Mousavi MF (2018) A dual Ni/Co-MOF-reduced graphene oxide nanocomposite as a high performance supercapacitor electrode material. Electrochim Acta 275:76-86

49. Wen P, Gong PW, Sun JF, Wang JQ, Yang SR (2015) Design and synthesis of Ni-MOF/CNTs composites and $\mathrm{rGO} /$ carbon nitride composites for an asymmetric supercapacitor with high energy and power density. J Mater Chem A 3:13874-13883

50. Zheng K, Li G, Xu C (2019) Advanced battery-supercapacitor hybrid device based on $\mathrm{Co} / \mathrm{Ni}$-ZIFs derived $\mathrm{NiCO}_{2} \mathrm{~S}_{4}$ ultrathin nanosheets electrode with high performance. Appl Surf Sci 490:137-144

51. Lee DY, Shinde DV, Kim E-K, Lee W, Oh I-W, Shrestha NK, Lee JK, Han S-H (2013) Supercapacitive property of metal-organicframeworks with different pore dimensions and morphology. Microporous Mesoporous Mater 171:53-57

52. Rajak R, Saraf M, Mohammad A, Mobin SM (2017) Design and construction of a ferrocene based inclined polycatenated CoMOF for supercapacitor and dye adsorption applications. J Mater Chem A 5:17998-18011

53. Zhang SY, Yang ZD, Gong K, Xu B, Mei H, Zhang HB, Zhang JX, Kang ZX, Yan YG, Sun DF (2019) Temperature controlled diffusion of hydroxide ions in 1D channels of Ni-MOF-74 for its complete conformal hydrolyzing to hierarchical $\mathrm{Ni}(\mathrm{OH})_{2}$ supercapacitor electrodes. Nanoscale 11:9598-9607

54. Sun SY, Huang MJ, Wang PC, Lu M (2019) Controllable hydrothermal synthesis of $\mathrm{Ni} / \mathrm{Co} \mathrm{MOF}$ as hybrid advanced electrode materials for supercapacitor. J Electrochem Soc 166:A1799-A1805

55. He SH, Li ZP, Wang JQ, Wen P, Gao JC, Ma LM, YangZG YSR (2016) MOF-derived $\mathrm{Ni}_{x} \mathrm{Co}_{1-x}(\mathrm{OH})_{2}$ composite microspheres for highperformance supercapacitors. RSC Adv 6:49478-49486

56. Yi H, Wang HW, Jing YT, Peng TQ, Wang XF (2015) Asymmetric supercapacitors based on carbon nanotubes@NiO ultrathin nanosheets core-shell composites and MOF-derived porous carbon polyhedrons with super-long cycle life. J Power Sources 285:281-290

57. Wang J, Zhong Q, Xiong YH, Cheng DY, Zeng YQ, Bu YF (2019) Fabrication of 3D Co-doped Ni-based MOF hierarchical microflowers as a high-performance electrode material for supercapacitors. Appl Surf Sci 483:1158-1165

Publisher's Note Springer Nature remains neutral with regard to jurisdictional claims in published maps and institutional affiliations. 